\title{
Adaptación de una metaheurística evolutiva para generar árboles enrutadores en una red de sensores inalámbricos del contexto de la agricultura de precisión ${ }^{*}$
}

\author{
Ángela María Rodríguez** \\ Juan Carlos Corrales ${ }^{* * *}$
}

Recibido: 28/07/2015 - Aceptado: 02/05/2016

DOI: $10.22395 /$ rium.v15n29a4

\begin{abstract}
Resumen
Las redes de sensores inalámbricos (WSN, por sus siglas en inglés) son ampliamente usadas para monitorizar variables de interés en fenómenos como campos de cultivo, contexto en el cual la inclusión de una WSN fortalece el quehacer de la agricultura de precisión. En este campo de aplicación surgen retos de diseño para las redes como la necesidad de alcanzar una longevidad de los nodos sensores, (que sirven como fuente de datos) de por lo menos seis meses, en correspondencia con el período de sembrado. Trabajos previos afirman que la técnica de enrutamiento usada en una red de sensores inalámbricos es un factor de alta incidencia en su longevidad. En este artículo se presenta MOR4WSN, una propuesta de enrutamiento que surge de la adaptación de un algoritmo genético multi-objetivo. A partir de despliegues de WSN, MOR4WSN crea topologías con estructura jerárquica que permitan un enrutamiento apropiado preservando la longevidad de la red. Los resultados actuales muestran resultados prometedores.
\end{abstract}

Palabras clave: agricultura de precisión, algoritmos evolutivos, optimización multi-objetivo, redes de sensores inalámbricos.

\footnotetext{
La presente propuesta estuvo apoyada por el Programa para el Fortalecimiento de la Red Interinstitucional de Cambio Climático y Seguridad Alimentaria - RICCLISA; específicamente por el proyecto Servicios de generación de alertas Agroclimáticas como soporte a la toma de decisiones del sector Cafetero Colombiano - AgroCloud. Dicho proyecto es financiado por la Universidad del Cauca, el CINARA, CENICAFE, CIAT, CREPIC y el programa de Redes de Conocimiento de COLCIENCIAS para el periodo 2013-2017

** Msc. Ingeniería Telemática. Docente investigadora en la Fundación Universitaria de Popayán. C11. 53 N. ${ }^{\circ}$ 5-21, Popayán.+57 3016900224. angela.rodriguez@docente.fup.edu.co

*** Phd. Ciencias de la Computación. Director del Grupo de Investigación GIT, Universidad del Cauca. Crr. $2^{\mathrm{a}}$ N.3N-111, sector Tulcán, Popayán.+57 3004814686. jcorral@unicauca.edu.co
} 


\title{
Adapting an evolutionary metaheuristic to generate routing trees in a wireless sensor network in the context of precision agriculture
}

\begin{abstract}
Wireless sensor networks (WSN) are widely used to monitor variables of interest in phenomena such as crop fields. In such a context the inclusion of a WSN strengthens the task of precision agriculture. For networks used in precision agriculture design challenges have emerged, like the need to achieve longevity for sensor nodes (serving as a data source), of not less than six months, corresponding to the cultivation periods. Previous works say that the routing technique used in wireless sensor networks is a high incidence factor in its longevity. In this paper, MOR4wSN, a routing proposal coming from the adaptation of a multi-objective genetic algorithm (MOGA) approach is presented. MOR4WSN creates tree structures from wsn deployment for proper routing to preserve the longevity in a WSN. Preliminary evaluation shows promising results.
\end{abstract}

Key words: precision agriculture, evolutionary algorithms, multi-objective optimization, wireless sensor networks. 


\section{INTRODUCCIÓN}

Una red de sensores inalámbricos (WSN, por sus siglas en inglés) es un conjunto de dispositivos semejantes a un microcomputador distribuidos espacialmente en un área de interés, conocidos como nodos sensores; estos poseen unidad de memoria, procesamiento, radiofrecuencia y sensado, y una fuente de energía limitada. Los nodos sensores capturan, a través de su módulo de sensado, un conjunto de valores para cada parámetro de interés en el ambiente circundante, cuyos valores son enviados por el medio inalámbrico, en forma de paquetes de datos, hacia una estación base centralizada que no tiene limitaciones de energía [1,2]. Entre las aplicaciones de despliegue de las WSN se destacan las construcciones civiles, ambientes de hogar, de salud, y los campos de cultivo. Este último contexto se conoce como agricultura de precisión.

La agricultura de precisión es un concepto de gestión de parcelas agrícolas que estudia la variabilidad de suelo y clima entre diferentes campos de cultivo con el fin de ajustar a la medida la aplicación de insumos como agua, fertilizantes, pesticidas, etc., $\mathrm{y}$, consecuentemente, incrementar los niveles de productividad [3].

Una WSN usada en agricultura de precisión monitoriza la evolución del producto desde la siembra hasta la cosecha, lo cual implica un requerimiento en la longevidad de la red de por lo menos seis meses, que corresponde a dicho período [4].

La longevidad de una red de sensores se define como el período de tiempo que transcurre desde el inicio de operación de la red hasta que un nodo (o un porcentaje de ellos) es incapaz de enviar sus datos a la estación base porque se ha descargado su batería [1].

Así, incrementar el ciclo de vida de una WSN se convierte en un reto de diseño porque: (i) los campos de cultivo en países en vía de desarrollo generalmente están ubicados en lugares montañosos y remotos, dificultando así recargar las baterías de los nodos sensores, (ii) la extensión de las parcelas está en términos de hectáreas, lo que significa que los paquetes de datos deben realizar recorridos extensos por la red hasta llegar a su destino: la estación base, y (iii) en una WSN la fase de transmisión de datos produce el mayor consumo energético debido al uso del módulo de radiofrecuencia; este consumo, a su vez, se ve afectado por la distancia del enlace de transmisión, entre otros parámetros.

En este sentido, Bari y sus colegas afirman [5]: "el tiempo de vida de una WSN puede variar considerablemente dependiendo del esquema de enrutamiento usado", mientras que en [6] se plantea: "la función de un protocolo de enrutamiento en una WSN es generar las vías para comunicar los dispositivos que se encuentran distribuidos en la red". 
En una etapa preliminar de esta propuesta, documentada en [7], se listaron los parámetros de influencia en la fase de transmisión de datos en una WSN aplicada a la agricultura de precisión, y se definió la función de aptitud para solucionar el enrutamiento a través de un algoritmo genético simple.

En el presente artículo se propone MOR4WSN (Multi-Objective Routing for Wireless Sensor Networks), una aproximación de optimización multi-objetivo para seleccionar las rutas de transmisión de los datos recolectados desde los nodos fuente hasta la estación base en una WSN desplegada en un ambiente agrícola, de tal manera que dichas rutas presenten ventajas para la longevidad de la red.

MOR4WSN adapta el NSGA-II para generar estructuras de enrutamiento de WSN con topología jerárquica. Las pruebas de simulación permiten comparar los efectos sobre el ciclo de vida de una WSN al utilizar MOR4WSN como su técnica de enrutamiento, frente a una técnica tradicional como lo es Tree Routing de Zig Bee.

El resto de este artículo se organiza de la siguiente manera: la sección 1 presenta el estado del arte con un marco teórico y los trabajos relacionados, la sección 2 describe la adaptación algorítmica propuesta, la sección 3 muestra resultados de la experimentación, la sección 4 presenta las conclusiones y, finalmente, la sección 5 agradece a los entes financiadores de esta investigación.

\section{ESTADO DEL ARTE}

\section{A. Definiciones previas}

1) Algoritmo genético. El algoritmo genético inicia con un conjunto de posibles soluciones generadas aleatoriamente (población inicial) denominadas cromosomas (o individuos) que constan de un arreglo de genes siempre con igual longitud (ver figura 1).

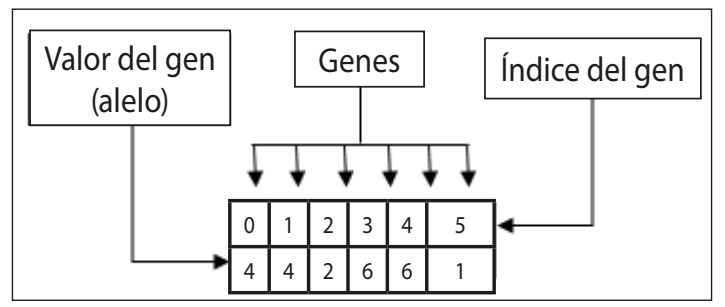

Figura 1. Cromosoma de 6 genes Fuente: elaboración propia

El algoritmo genético dispone de una función de aptitud que asigna una valoración a cada individuo, basado en qué tan cerca está de la solución óptima. La selección per- 
mite crear descendencia a partir del proceso de cruza, mientras que los hijos creados experimentan la mutación; esto implica alterar genéticamente la generación actual con el fin de mejorarla respecto a los criterios que se están optimizando [8].

2) Algoritmo genético multi-objetivo. Las aproximaciones multi-objetivo buscan solución a un problema que involucra la optimización de varios objetivos en paralelo y normalmente contrapuestos. Un algoritmo genético multi-objetivo ( $M O G A$, por sus siglas en inglés) modifica la forma como se calcula la aptitud de los individuos respecto al genético simple, siendo necesario definir un conjunto de funciones objetivo.

Por su naturaleza multi-objetivo, un MOGA no busca una única mejor solución, sino que produce un conjunto de soluciones, conocido como el conjunto de Pareto (o conjunto no dominado), refiriéndose a aquellas mejores soluciones de compromiso considerando todos los objetivos en juego.

Ejemplos de MOGA son: algoritmo genético de vector evaluado (VEGA, por sus siglas en inglés), algoritmo genético de nichos de Pareto (NPGA, por sus siglas en inglés) y algoritmo genético de clasificación no dominada (NSGA, por sus siglas en inglés). Estos algoritmos tienen complejidad computacional de $\boldsymbol{0}\left(m N^{\beta}\right)$, donde $m$ es el número de objetivos y $N$ es el tamaño de la población.

3) NSGA-II. Una evolución del NSGA es el NSGA-II (Fast Elitist Non Dominated Sorting Genetic Algorithm) propuesto por Kalyanmoy Deb en 2002 [9]. El NSGA-II se ocupa de mantener diversidad entre las soluciones de la población, gracias a la incorporación de los parámetros sharing y crowding distance, logrando reducir su complejidad a $\boldsymbol{O}\left(\mathrm{mN}^{2}\right)$. Una explicación detallada del funcionamiento del NSGA-II se puede consultar en [10].

\section{B. Trabajos relacionados}

Para la generación de la población inicial existe la condición de que los genes (nodos) consecutivos no pueden exceder una distancia umbral [11], mientras que la selección de un nodo de próximo salto depende de la distancia del enlace, del consumo de energía promedio del camino, y de la distancia del nodo actual hasta la estación base. Para la mutación se comparte información global de la población, gracias a la existencia de un registro histórico del mejor cromosoma (aquel con máximo valor de aptitud hasta el momento). En [12] la función de aptitud la determina la energía residual de cada individuo y su carga de transmisión y recepción; se entiende por carga la energía requerida para recibir los paquetes entrantes de sus hijos y transmitirlos a su padre.

Por su parte, Bari y sus colegas [5] definen la función de aptitud de acuerdo con el tiempo de vida de la red en términos de rondas. Para la operación de mutación 
seleccionan, de entre los nodos relé, un nodo crítico que disipa la máxima energía, y se reemplaza el nodo de próximo salto de este nodo crítico por un nuevo nodo relé de próximo salto, o se desvía algún flujo de entrada de ese nodo crítico, hacia otro nodo relé.

En GAR [13], la función de aptitud se construye de manera que la distancia total cubierta por los nodos es minimizada en cada ronda. Para la mutación, de manera similar a [14], se selecciona un nodo crítico que en este caso es el gen que contribuye con la máxima distancia en una ronda, y lo reemplazan por otro nodo que conduzca a la estación base cubriendo una menor distancia.

De las anteriores aproximaciones, ninguna ha orientado la solución hacia un área de aplicación específica; esto permite que los parámetros incluidos en la función de aptitud se elijan de manera indiscriminada. Además, todas las propuestas trabajan con el algoritmo genético estándar cuya función de aptitud no tiene en cuenta la contradicción que puede existir al optimizar varios parámetros simultáneamente.

MOR4WSN adapta el NSGA-II de manera que las funciones objetivo corresponden con variables extraídas de las características de las WSN desplegadas en campos de cultivo.

\section{MOR4WSN}

Esta sección presenta cada componente del NSGA-II que debe ser adaptado para convertirse en MOR4WSN; se presentan sus dificultades de implementación y propuesta de solución.

\section{A. Modelo dered}

En los proyectos documentados en [16-20] se ejecutaron despliegues de WSN en campos de cultivo con diferentes productos agrícolas. Del análisis de estos trabajos se define el modelo de red para MOR4WSN como sigue.

1) Los nodos sensores se despliegan de manera aleatoria y son estacionarios después del despliegue.

2) La ubicación de los nodos es conocida.

3) Cada período de recolección y transmisión de datos es referido como una ronda.

4) Durante una ronda, la estación base recibe datos de todos los nodos; cada sensor adquiere las muestras de los datos requeridos para su entorno, agrega algún paquete de entrada desde sus vecinos y lo reenvía a su padre o a la estación base. 
5) Un enlace inalámbrico se establece entre dos nodos solo si están dentro del mismo rango de comunicación (nodos vecinos).

6) La red es homogénea, es decir, que para todos los nodos la energía inicial es la misma y su rango de cobertura es el mismo.

\section{B. Representación de la solución}

En MOR4WSN la representación del cromosoma se define como en los trabajos previos [11-15]; el cromosoma representa un árbol de enrutamiento donde cada nodo está etiquetado con un número entero de 0 a $N$, siendo $N$ la cantidad de nodos, como se observa en la figura 2a. En la figura $2 \mathrm{~b}$ el valor del gen en la posición 0 es 2, indicando que el nodo 0 selecciona al nodo 2 para transmitirle sus datos; el nodo 2 transmite hacia el nodo 5 , y el valor en 5 es 6 , indicando que el próximo nodo es la estación base. Por lo tanto la ruta completa de recolección de los datos del nodo 0 se expresa como el camino $0 \rightarrow 2 \rightarrow 5 \rightarrow 6$.

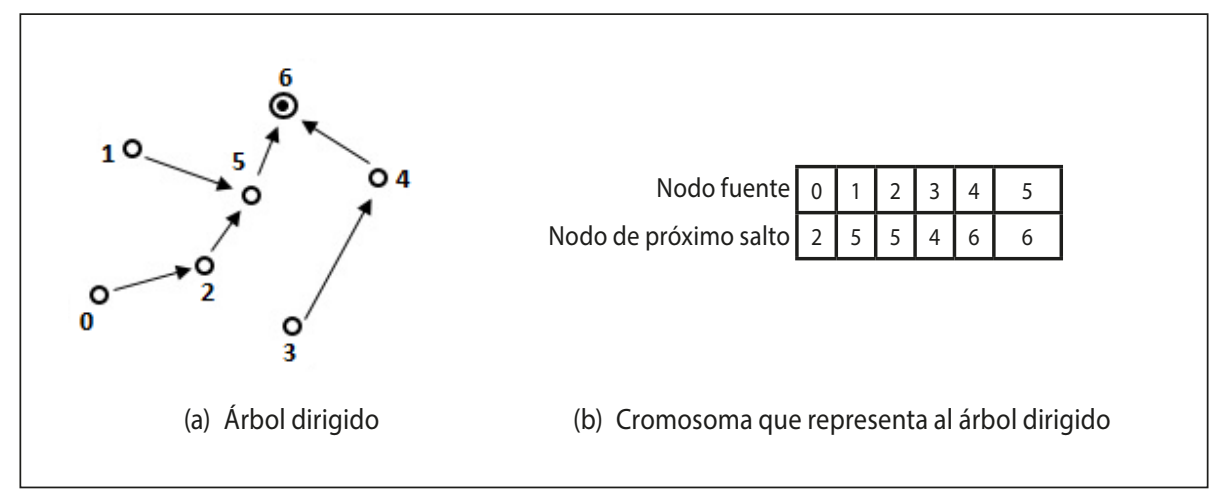

Figura 2. Solución de enrutamiento de una WSN

Fuente: elaboración propia

\section{Funciones objetivo}

Cuando en una red jerárquica como la de la figura 2a existen nodos que reciben y retransmiten paquetes de datos de muchos otros nodos de la red (nodos enrutadores), desgastarán sus baterías más rápidamente que aquellos nodos que realizan pocas retransmisiones; es por esto que, de acuerdo con los resultados en [21], una red de monitorización con estructura de árbol es longeva en la medida que se disminuya su cantidad de nodos hoja y su profundidad. Lo anterior se puede sintetizar como la búsqueda de balanceo de carga en la red, que se entiende como equilibrar el consumo de energía entre todos los nodos de la red; esto es, hacer una distribución equitativa de paquetes de datos recibidos, procesados y enviados [2]. 
La figura 3 indica que reducir simultáneamente el valor de los parámetros nodos hoja y profundidad en un árbol de enrutamiento es una situación contradictoria. De este modo, un MOGA es una metaheurística adecuada para el problema de enrutamiento.

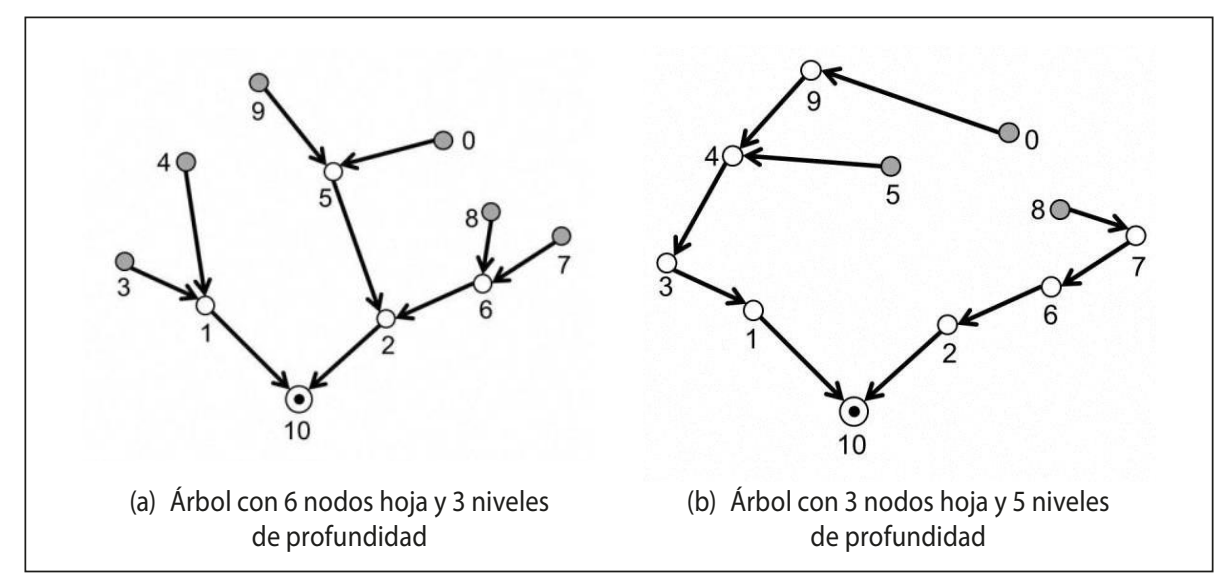

Figura 3. Árboles de enrutamiento en una WSN

Fuente: elaboración propia

Por otro lado, es pertinente tener en cuenta el consumo energético de cada estructura durante una ronda de sensado. La energía de transmisión y recepción consumida por cada nodo en una ronda de recolección de datos se modela con las ecuaciones (a) y (b) donde $k$ es el tamaño del paquete y $d$ la distancia de transmisión; $\mathbf{E}_{\text {elec }}$ es el coeficiente de energía eléctrica con la cual opera el radio, $\mathbf{E}_{\text {amp }}$ el coeficiente de energía del amplificador y $n$ es el coeficiente de pérdida de camino que generalmente toma valores entre 2 y 4.

$$
\begin{aligned}
& E_{T X}(k, d)=\left(\xi_{\text {elec }}+\xi_{\text {amp }} * d^{n}\right) * k \\
& E_{R X}(k)=\xi_{\text {elec }} * k
\end{aligned}
$$

Finalmente, las funciones objetivo en MOR4WSN son:

Función objetivo 1: Disminuir la cantidad de nodos hoja.

Función objetivo 2: Disminuir la profundidad del árbol.

Función objetivo 3: Disminuir la energía consumida en cada ronda de sensado.

\section{Población inicial}

En la implementación genérica del NSGA-II los cromosomas de la población inicial se forman aleatoriamente de manera que los alelos de cada individuo toman cualquier 
valor entre 0 y $N$ ( $N$ es la cantidad de nodos de la red), permitiendo a un índice tomar un valor de alelo que no necesariamente está en su lista de vecinos, es decir, un nodo de próximo salto con el cual no se tenga comunicación directa. Dicho individuo sería inválido dentro de la población de MOR4WSN; por lo tanto, se debe asegurar que todos los cromosomas de la población inicial representen cromosomas válidos para el problema de enrutamiento. Se identifican entonces los siguientes puntos por resolver:

i) En un cromosoma cada alelo debe representar a un nodo vecino de su correspondiente índice.

ii) La estación base debe estar incluida en el árbol y debe ser la raíz.

iii) Prevenir la formación de ciclos en el árbol.

En MOR4WSN cada gen se forma entonces de la siguiente manera: los números mínimo y máximo que requiere el NSGA-II para sortear el valor de un alelo se toman del rango entre 0 y la cantidad de vecinos del nodo fuente (índice).

\section{E. Cruza}

En la figura 4 es claro que el cruce de dos puntos puede generar hijos (topologías de enrutamiento) que no son árboles. En MOR4WSN es indispensable que todas las soluciones potenciales (cromosomas) sean árboles válidos para el enrutamiento.

Los puntos por resolver para MOR4WSN cada vez que se aplica el operador de cruza son:

i) Existencia de ciclos en el (los) hijo(s).

ii) La estación base debe aparecer en la estructura que representa al hijo.

\section{F. Mutación}

La figura 5 indica que la aplicación de la mutación con el NSGA-II puede dar lugar a un ciclo en la estructura resultante, o a la no existencia de la estación base. Además, para lograr una topología funcional después de la mutación de un individuo, es indispensable que el nuevo alelo sea vecino del índice alterado.

MOR4WSN preserva árboles en las generaciones alteradas por la mutación, a través del diseño algorítmico que resuelve los siguientes aspectos:

i) Prohibir la creación de ciclos en el hijo mutado.

ii) El nuevo nodo de próximo salto del nodo fuente mutado debe estar en su lista de vecinos. 

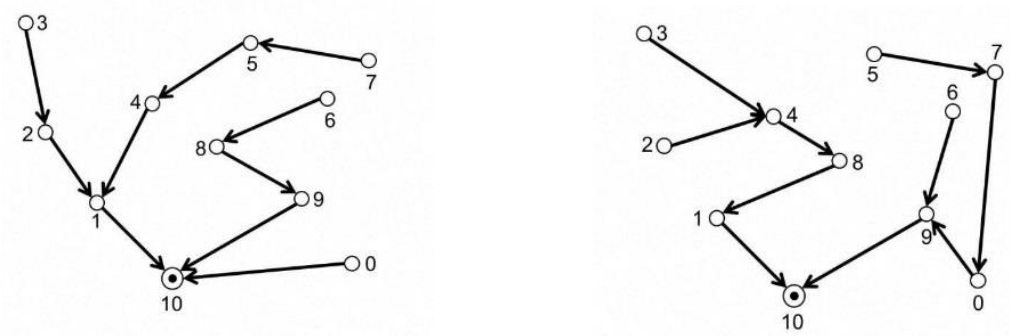

(a) Topología de los padres P1 y P2

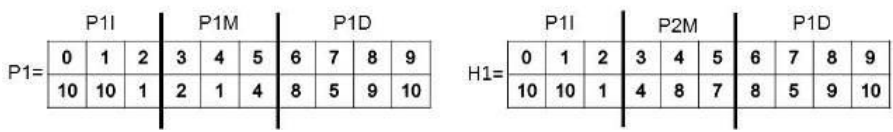

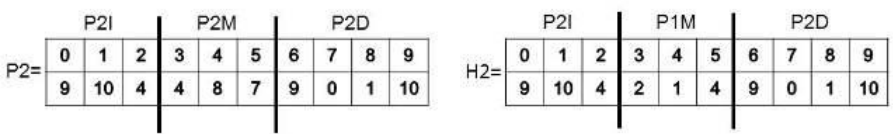

(b) Cruza entre $P 1$ y $P 2$

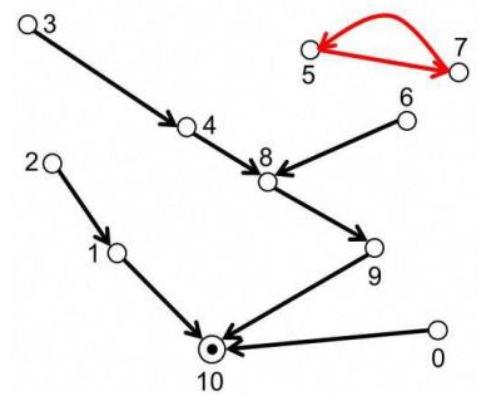

(c) Hijo $\mathrm{H} 1$ generado por la cruza entre $P 1$ y $P 2$

Figura 4. Cruza de dos puntos que genera un hijo con ciclo Fuente: elaboración propia

\section{G. Índice binario para seleccionar en el conjunto de Pareto}

Siendo una aproximación evolutiva multi-objetivo, MOR4WSN no brinda una única solución (una única topología de árbol de enrutamiento), sino un conjunto de soluciones conocida como el conjunto de Pareto, el cual tiene el mismo tamaño que la población.

El concepto de dominancia de Pareto se ilustra en la figura 6; aquí una solución $a$ 'domina' a $b$ siempre y cuando: $a$ no sea peor que $b$ en todos los objetivos, y $a$ sea estrictamente mejor que $b$ en por lo menos un objetivo. Así, el conjunto de soluciones 


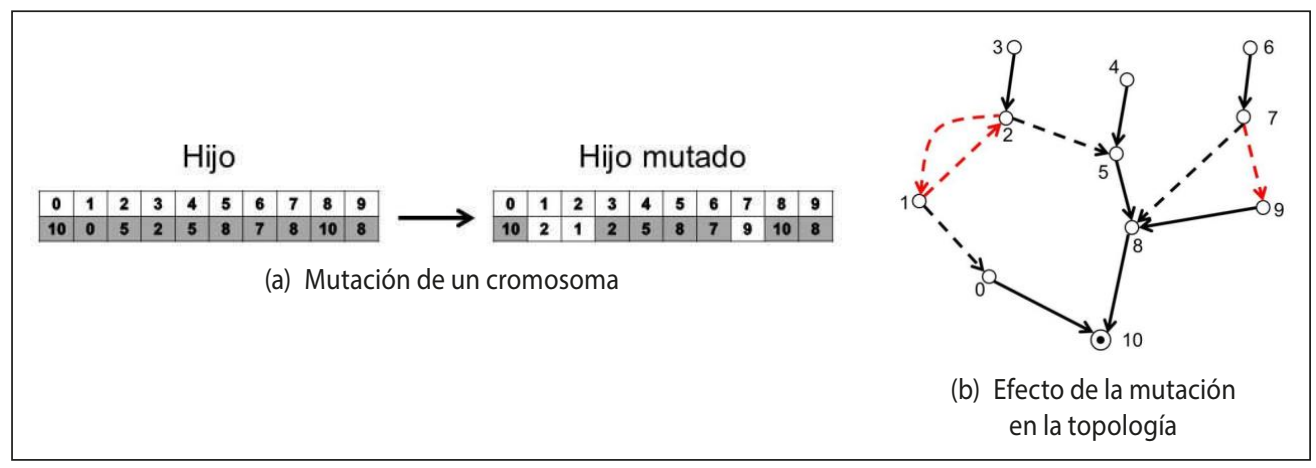

Figura 5. Mutación en NSGA-II

Fuente: elaboración propia

que no están dominadas por ninguna otra conforman el Frente no dominado también conocido como soluciones no dominadas de Pareto. En la figura 6 estas soluciones no dominadas están representadas por los puntos 3 y 5 .

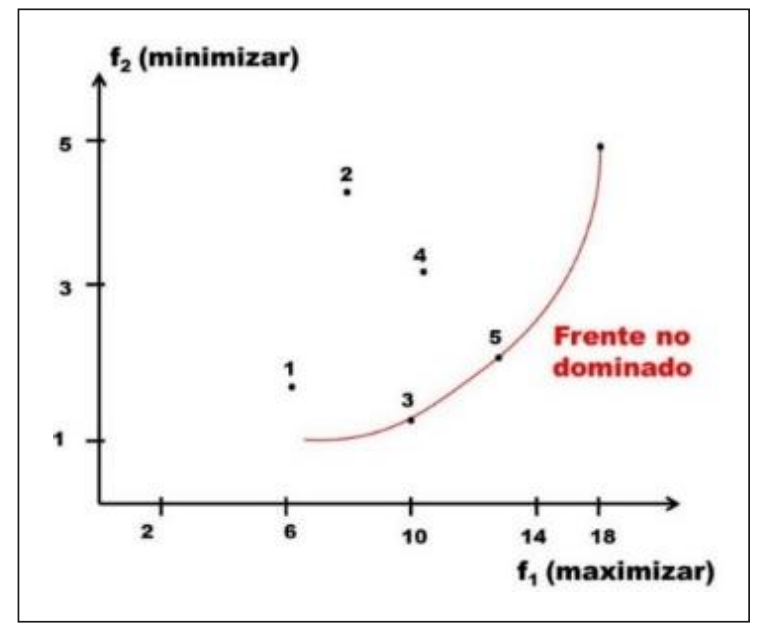

Figura 6. Dominancia de Pareto Fuente: [10]

En MOR4WSN el tamaño de la población toma valores del orden de los 100 individuos $(\mathrm{N}=100,200, \ldots)$, es decir, que la población optimizada respecto a las funciones objetivo brinda $\mathrm{N}$ soluciones entre las cuales un tomador de decisiones (conocedor del contexto) debe seleccionar una única topología de enrutamiento, es decir, un único árbol optimizado. Para seleccionarlo es necesaria la existencia de preferencias, las cuales usualmente no están disponibles, es decir, no existen criterios para definir si una solución del conjunto de Pareto es preferible a otra, o el conjunto de datos es inmanejable para el tomador de decisiones. 
Para seleccionar una solución multicriterio a posteriori en el conjunto de Pareto, algunos métodos (métodos outranking) llevan a cabo comparaciones por pares con el fin de establecer si existe preferencia, indiferencia o son incomparables. Sin embargo, estos métodos requieren que el tomador de decisiones establezca algunas ponderaciones [22].

En MOR4WSN un criterio para seleccionar solo una solución es evitar soluciones atípicas que a menudo están presentes en el conjunto de Pareto porque no están dominadas por ninguna otra solución en uno de los objetivos. De esta manera, las soluciones del conjunto de Pareto se comparan por pares a través de un índice binario que representa cada árbol de enrutamiento; usando este índice las soluciones atípicas quedan excluidas de ser seleccionadas como estructura de enrutamiento final.

En el proceso comparativo cada árbol del frente de Pareto se compara con una estructura atípica, y MOR4WSN selecciona el árbol que tiene menor similitud con dicha estructura, como se ilustra en la figura 7.

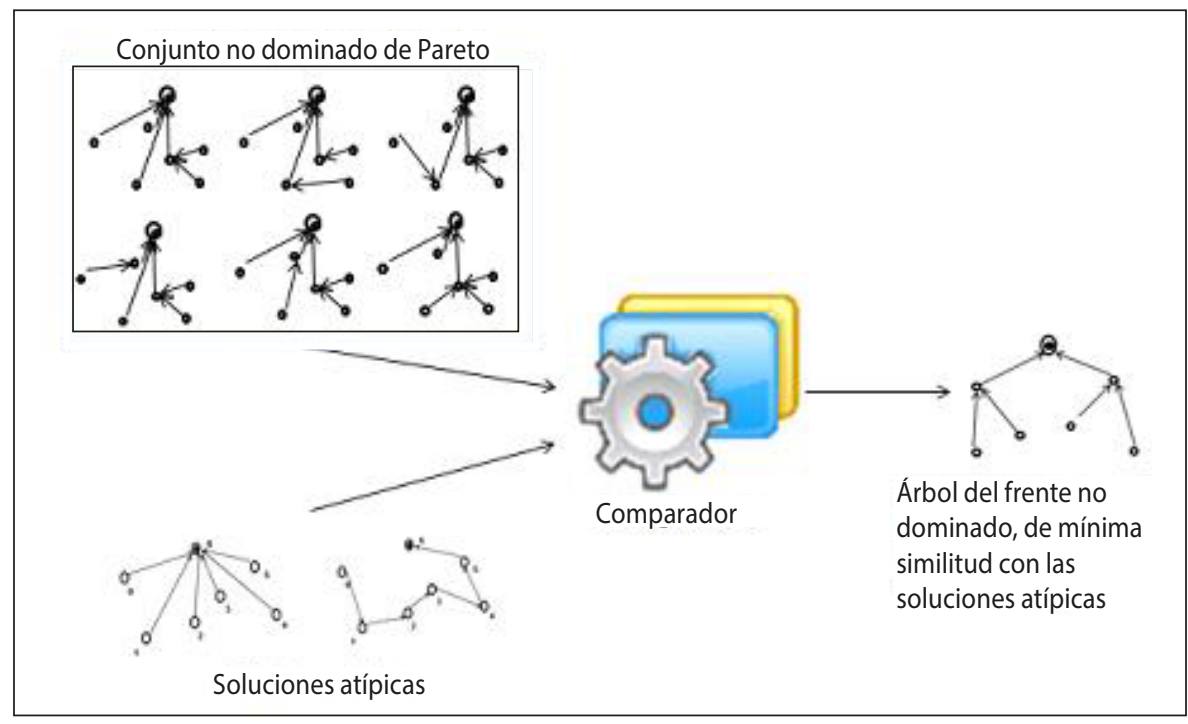

Figura 7. Comparador de grafos en MOR4WSN

Fuente: elaboración propia

El comparador de la figura 7 hace lo siguiente: Sean $G 1$ y $G 2$ los grafos etiquetados (que representan árboles de enrutamiento) que se compararán, siempre será $G 1$ la solución atípica mientras que $G 2$ es una solución no dominada no atípica como en la Figura 8a. Así, en la figura 8b están los cromosomas correspondientes: $G 1 \rightarrow C 1$ y $G 2 \rightarrow C 2$. 


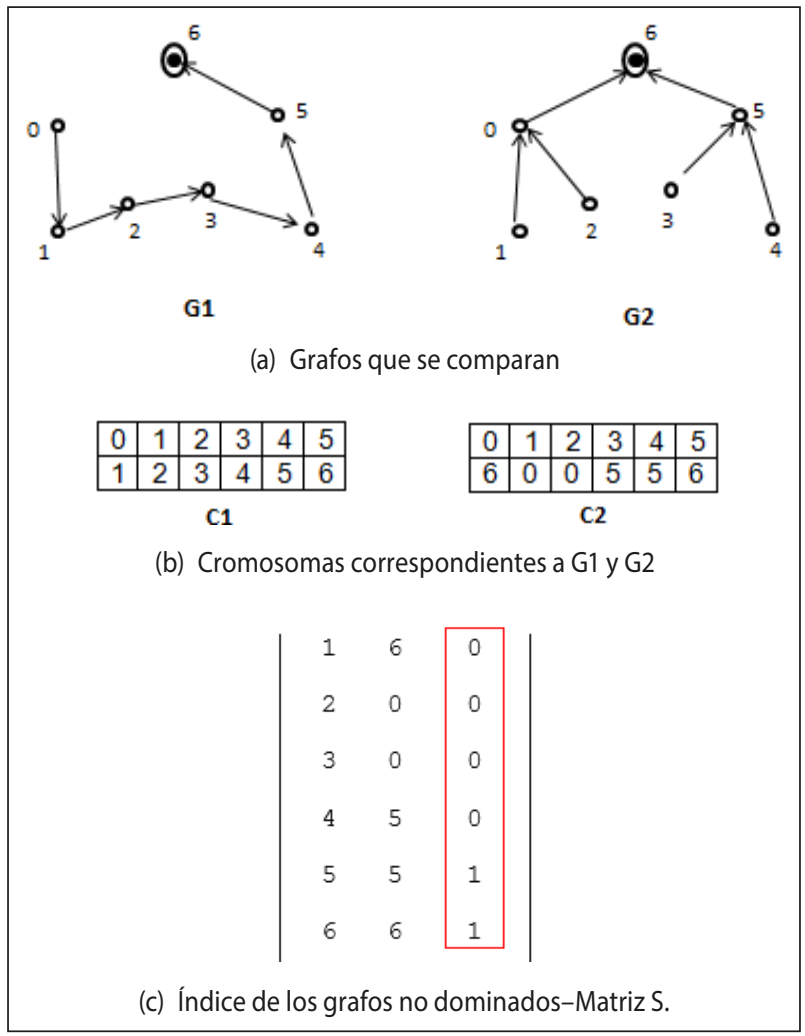

Figura 8. Proceso de comparación de grafos Fuente: elaboración propia

Partiendo de $C 1$ y $C 2$ se crea la matriz $S$ de tamaño $N$ x 3 como se muestra en la figura $8 . \mathrm{S}$ se crea de la siguiente manera:

$$
S=\left\{x_{i j} / x_{i o}=C 1, x_{i 1}=C 2, x_{i 3}=0 \text { si } x_{i 0}=x_{i 1} \text { o } x_{i 3}=1 \text { si } x_{i 0}^{1} x_{i 1}\right\},
$$

donde $i=0,1, \ldots, N$ y $J=0,1,2$

La columna $S_{i 3}$ subrayada en rojo en la figura 8c es un vector binario formado de las coincidencias de elementos en cada fila de $S$. Sea $i$ la fila en consideración, si los elementos $(i, 0)$ e $(i, l)$ de $S$ coinciden exactamente, el elemento en la posición $i$ de $S_{i 3}$ toma el valor de 1 , de otra forma será 0 . La matriz $S$ es el índice de MOR4WSN; cada grafo tiene asociado su propio índice toda vez que $S$ ordena la información necesaria para definir la similitud entre los grafos G1 y G2. Luego, con los elementos de la columna $\mathrm{j}=3$ del índice de cada grafo se establece una relación de similitud como sigue: 
Sea

$t=\left\{x_{i 3} / x_{i 3} \in S\right\}$ donde $i=0,1, \ldots, N$

$z=\sum_{k=1}^{N-1}\left(t_{k}\right)$, para cada $t=1$ y $p=\left(\frac{z}{N}\right) * 100 \%$

Para el ejemplo de la figura 8 se tiene: $N=6, t=\{0,0,0,0,1,1\}, z=2, p=33.33 \%$.

Lo anterior significa que el nivel de similitud entre $G 1$ y $G 2$ es del $33.33 \%$, valor que toma MOR4WSN como criterio de selección entre los elementos del conjunto de Pareto. A manera de tomador de decisiones el módulo del índice binario de MOR4WSN selecciona finalmente solo un árbol: aquel que tenga el menor valor de $p$ entre los $(N$ 2)*2 valores de $p$ calculados.

\section{EVALUACIÓN}

Las pruebas realizadas sobre esta versión de MOR4WSN permitieron determinar la duración del ciclo de vida alcanzado por una red usando enrutamiento con MOR4WSN y con Tree Routing* (TR). El ciclo de vida se mide en términos de las rondas de sensado alcanzadas en dos casos: (i) hasta que el primer nodo es incapaz de enviar sus datos a la estación base (el nodo muere), (ii) hasta que el $10 \%$ de los nodos mueren.

Los resultados se muestran en la figura 9; y permiten afirmar que, independientemente a la cantidad de nodos de la red, una aproximación multi-objetivo como MOR4WSN da lugar a una mayor cantidad de rondas de sensado que cuando se usa un enrutamiento mono-objetivo como Tree Routing.

Cuando toma como referencia de longevidad la muerte del $10 \%$ de los nodos de la red, MOR4WSN supera en el $95 \%$ de los casos (diferentes cantidades de nodos) el ciclo de vida alcanzado respecto al uso de Tree Routing, como lo indica la figura 8b.

\section{CONCLUSIONES}

En este artículo se modelan los elementos de las WSN en los algoritmos genéticos, y se definen los problemas de implementación que surgen con cada operador/componente del NSGA-II durante la adaptación para representar árboles de enrutamiento válidos.

La optimización del consumo de energía es fundamental para la implantación de redes de sensores inalámbricos en contextos como la agricultura de precisión; esta optimización de energía a través de un enfoque evolutivo multi-objetivo se reflejó en

Protocolo de enrutamiento usado en el estándar de comunicación ZigBee, para redes con topología de árbol. 


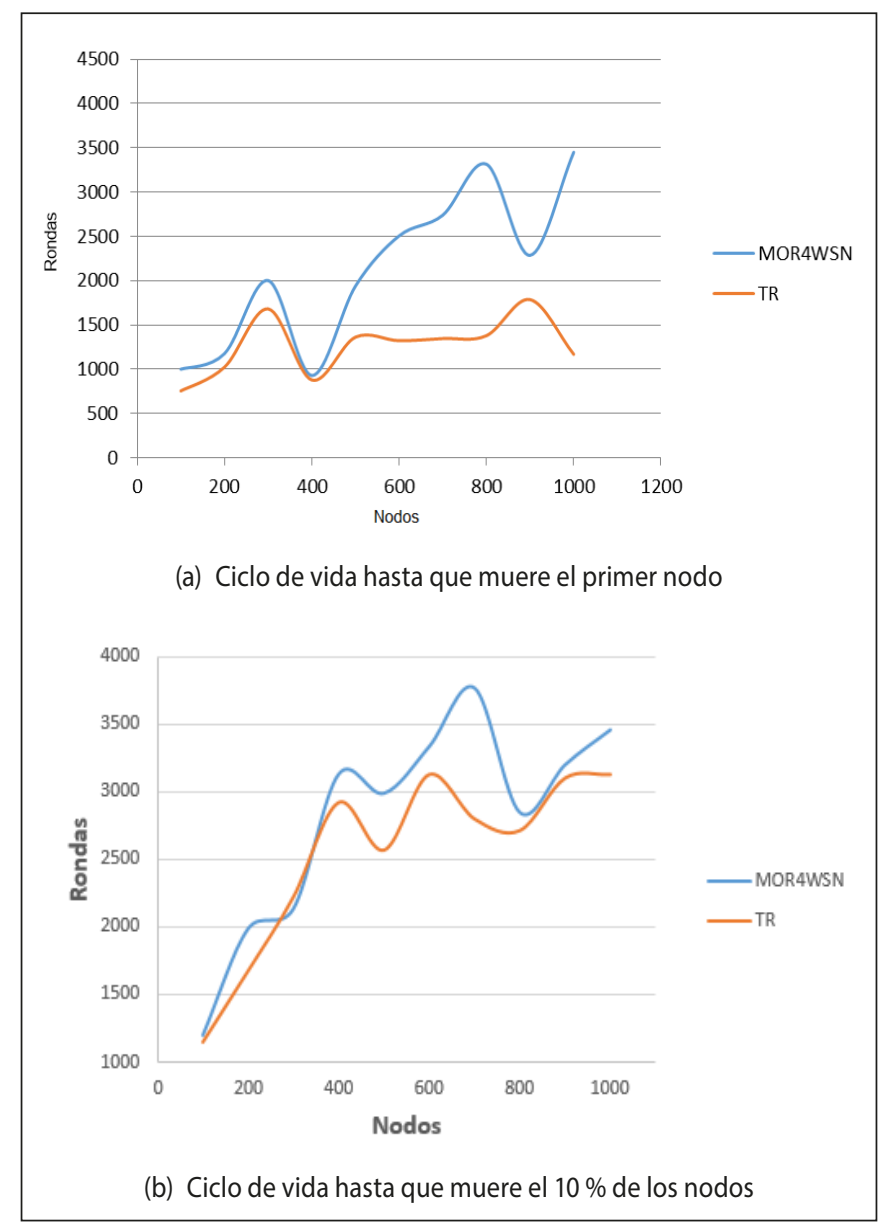

Figura 9. Tiempo de vida de la WSN Fuente: elaboración propia

un aumento de longevidad como lo indican las gráficas de la figura 8. En el momento se están realizando repetidas ejecuciones de las pruebas para determinar si hay alguna razón funcional para los picos presentados en las curvas de las figuras 8 a y 8b cuando la red tiene alrededor de 400, 700 y 900 nodos, o si se debe a algún ajuste en los parámetros de simulación.

\section{AGRADECIMIENTOS}

Los autores expresan agradecimiento a la Universidad del Cauca, a Colciencias y al proyecto AgroCloud de la Red Interinstitucional de Cambio Climático y Seguridad Alimentaria de Colombia (RICCLISA) por soportar esta investigación. 


\section{REFERENCIAS}

[1] P. Rawat, K. D. Singh, H. Chaouchi, and J. M. Bonnin, "Wireless sensor networks: a survey on recent developments and potential synergies", J. Supercomput., vol. 68, N. 1, pp. 1-48, Apr. 2014.

[2] X. Cui and Z. Liu, "BCEE: a balanced-clustering, energy-efficient hierarchical routing protocol in wireless sensor networks", in Network Infrastructure and Digital Content, 2009. IC-NIDC 2009. IEEE International Conference on, 2009, pp. 26-30.

[3] D. Emmen, "La agricultura de precisión: una alternativa para optimizar los sistemas de producción”, Investig. Pensam. Crít., Vol. 2, pp. 68-74, 2004.

[4] D. Villón Valdiviezo, “Diseño de una red de sensores inalámbrica para agricultura de precisión/ Daniel Villón Valdivieso”, 2011.

[5] A. Bari, S. Wazed, A. Jaekel, and S. Bandyopadhyay, "A genetic algorithm based approach for energy efficient routing in two-tiered sensor networks", Ad Hoc Netw., vol. 7, N. ${ }^{\circ}$, pp. 665-676, Jun. 2009.

[6] C. C. Nayibe, T. B. D. Alexander, A. Z. L. Cristina, and C. O. L. Miguel, "Redes de Sensores Inalámbricos".

[7] A. M. Rodríguez-Vivas, J.C. Corrales-Muñoz, "Adaptación del Algoritmo Genético para la Creación de Árboles de Enrutamiento en una Red de Sensores Inalámbricos", Lámpsakos, N. ${ }^{\circ} 10$, pp. 34-42, 2013.

[8] A. León Javier, "Diseño e implementación en hardware de un algoritmo bio-inspirado", Instituto Politécnico Nacional, México D. F., 2009.

[9] K. Deb, S. Agrawal, A. Pratap, and T. Meyarivan, "A fast elitist non-dominated sorting genetic algorithm for multi-objective optimization: NSGA-II”, Lect. Notes Comput. Sci., Vol. 1917, pp. 849-858, 2000.

[10] K. Deb, A. Pratap, S. Agarwal, and T. Meyarivan, "A fast and elitist multiobjective genetic algorithm: NSGA-II”, Evol. Comput. IEEE Trans. On, Vol. 6, N. 2, pp. 182-197, 2002.

[11] O. Islam, S. Hussain, and H. Zhang, "Genetic algorithm for data aggregation trees in wireless sensor networks", 2007.

[12] A. Chakraborty, S. Kumar, and M. Kanti, "A genetic Algorithm Inspired Routing Protocol for Wireless Sensor Networks", International Journal of Computational Intelligence Theory and Practice, vol. 6 N. $.^{\circ} 1,2011$.

[13] S. K. Gupta, P. Kuila, and P. K. Jana, "GAR: An energy efficient ga-based routing for wireless sensor networks", in Distributed Computing and Internet Technology, Springer, 2013, pp. $267-277$.

[14] A. Bari, S. Wazed, A. Jaekel, and S. Bandyopadhyay, "A genetic algorithm based approach for energy efficient routing in two-tiered sensor networks", Ad Hoc Netw., Vol. 7, N. 4 , pp. 665-676, Jun. 2009. 
[15] I. Apetroaei, I.-A. Oprea, B.-E. Proca, and L. Gheorghe, "Genetic algorithms applied in routing protocols for wireless sensor networks", 2011, pp. 1-6.

[16] S. Yoo, J. Kim, T. Kim, S. Ahn, J. Sung, and D. Kim, “A 2 S: automated agriculture system based on WSN", 2007, pp. 1-5.

[17] N. P. Karthickraja, V. Sumathy, and J. Ahamed, "A novel hybrid routing protocol for data aggregation in agricultural applications", 2010, pp. 227-231.

[18] A. H. Kabashi and J. M. H. Elmirghani, "A Technical Framework for Designing Wireless Sensor Networks for Agricultural Monitoring in Developing Regions”, 2008, pp. 395-401.

[19] J. Panchard, S. Rao, J.-P. Hubaux, and H. S. Jamadagni, "COMMONSense Net: A Wireless Sensor Network for Resource-Poor Agriculture in the Semiarid Areas of Developing Countries”. Inf. Technol. Int. Dev., vol. 4, N. ${ }^{\circ}$ 1, 2007.

[20] J. Hu, L. Shen, Y. Yang, and R. Lv, "Design and implementation of wireless sensor and actor network for precision agriculture", 2010, pp. 571-575.

[21] A. M. Ortiz Torres, “Técnicas de enrutamiento inteligente para redes de sensores inalámbricos”. Tesis doctoral. Universidad de Castilla La Mancha. Albacete-España, 2011.

[22] A. Rodríguez, A. Ordóñez, A. Ordóñez, "Energy consumption optimization for sensor networks in the IoT", IEEE Colombian Conference on Communications and Computing, 2015. 
